\title{
An Assessment of Lower Respiratory Tract Infections in Paediatrics-A Seasonal Study
}

\author{
Nisha Rajan', Binai Kochunni Sankar ${ }^{1}$, Prasad Sivanna Mruthyunjaya ${ }^{2}$ \\ ${ }^{1}$ Department of Pharmacy Practice, Acharya and B. M. Reddy College of Pharmacy, Bangalore, INDIA. \\ ${ }^{2}$ Department of Paediatrics, Dr. B. R. Ambedkar Medical College and Hospital, Bangalore, INDIA.
}

\begin{abstract}
Introduction: Paediatrics are a group of population vulnerable to many infections. Among these infections, the burden of RTls are growing faster day by day because of the poor lifestyle, polluted environment and even inadequate care given to them when they are born. LRTIs have been affecting the quality of life of a number of children which can even turn fatal. Early detection, treatment and prevention can improve the later life of a child. At the same use of therapeutic agents in such infections need to be studied. Antibacterials have been playing a very important role since decades. Hence judicious use of such agents are necessary to make sure that, this special age group would not develop any resistance in future. Objective: To study the incidence of LRTI and use of antibacterials in paediatrics. Methodology: This is a prospective observational study carried out over a period of three months among In-patients and Out-patients in the department of Paediatrics. The patient's bystanders were briefed about the study and consent was obtained prior to their enrollment. All the patients visiting the $\mathrm{OP}$ as well as admitted to the hospital being treated with a single or multiple antibacterial agent were reviewed on a daily basis by the clinical pharmacist. All the documented data were evaluated by applying appropriate statistical methods. Result: A total of 80 patients were enrolled in which $92.5 \%$ were found be in the age range of 0 to 5 years. Males $(57.5 \%)$ were found to be affected more than females. The number of patients in IP was more, $56.25 \%$. In the month of February, $86 \%$ of the patients were seen visiting OP whereas in December $67 \%$ were admitted to the Hospital. It was found that $98 \%$ of the cases were caused by bacteria. Most of them, $(80 \%)$ received antibacterial therapy. Amoxicillin-clavulanic acid combination was most $(37.5 \%)$ commonly used and injectable were preferred. Supportive therapy was also given, and maximum patients, $27.70 \%$ were on nebulization. Conclusion: This study concludes that children below the age of 5 years are greatly vulnerable to LRTI. The number of cases increased as the temperature decreased. Combination of antibacterials are preferred in the present scenario which show the development of resistance. Hence, rational prescribing of antibacterials is necessary. Moreover, culture tests are to be performed prior to the initiation of therapy so that unwanted use can be controlled. The Paediatricians should strictly stick to the guidelines so that quality of life of children can be improved.
\end{abstract}

Key words: Antibacterials, Paediatrics, LRTI, Supportive therapy, Culture tests, Temperature fluctuations.

\section{INTRODUCTION}

Paediatrics are the special group of population whose age limit usually ranges from birth to 18 years. They are found to be affected with many infectious diseases world-wide. The young child, whether well or ill, needs care to survive. The care of a child requires an understanding of their particular stage of growth and development and their individual ways of thinking, feeling and coping with the environment. The immature body organs as well as reduced immunity paves way for a number of infectious diseases which not only affects their growth and development but also the quality of life, later on.

Submitted date : $12 / 12 / 2015$

Accepted date : 22/12/2015

DOI: 10.5530/ijopp.9.1.9

Address for correspondence: Nisha Rajan, Department of Pharmacy Practice, Acharya and B. M. Reddy College of Pharmacy, Bangalore, INDIA.

Phone no: 9620454173

E-mail:nisharajan072@gmail.com

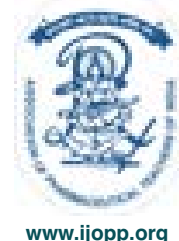

www.ijopp.org 
A lower respiratory tract infection is an infection that affects the trachea or windpipe and lungs, which include;

- Bronchitis (infection of the large airway or bronchi)

- Bronchiolitis (infection of the small airways or bronchioles)

- Croup (infection of the trachea or windpipe in children)

- Influenza (widespread infection of the upper and lower respiratory tract including the nose, throat and occasionally, bronchi and lungs)

- Pneumonia (infection of the alveoli and surrounding lung tissue $)^{1}$

According to a survey in 2000 , the number of prescription for antibacterials for children were half that in 1996, with amoxicillin counting for 50\%. There was a marked regional variation in prescribing rates. The survey also depicted that in 1992, around $50 \%$ of bacteremia were due to Gram-Positive bacteria, but this has increased to over $75 \%$ in 2003 with Staphylococcus aureus now becoming the leading significant bacteraemia reported in children. ${ }^{2}$

In 2010, it was found that specific viral single and co-infections as well as viral load contribute to disease severity in children with LRTIs. ${ }^{3}$

Since the Indian population has a low life expectancy, its most pressing health problems are those that affect infants and children, principally accidents, gastrointestinal diseases, tuberculosis, respiratory diseases and so on. The problem of infant and child health results from the high incidence and severity of infectious diseases, delay in obtaining treatment resulting in secondary complications and difficulty in providing preventive medical services. ${ }^{4}$ Today the world has got most sophisticated methods for detection and identification of the use of antibiotic therapy. Polymerase chain reaction (PCR) rapid molecular diagnostic assay can be a promising method in timely detection of bacterial pathogen. Adherence to national guidelines and clinical outcome data as well as continuous audit process is required to eliminate such variations that are growing day by day.

\section{Need for the study}

Respiratory health is a major issue among children and adolescents. Although genetic factors play an important role in an individual's risk of developing LRTI like asthma, these factors probably do not account large difference among population. Several childhood factors are found to be significantly associated with risk, such as, not being of high socioeconomic status, living in crowded household, living in polluted areas, children being breastfed for less than 9 months and so on. A mixture of different conditions with distinct etiologies and risk factors combine to cause childhood wheezing. Therefore, it is important to study the presence of wheezing among individuals. Understanding the role of social, environmental and behavioral factors in children are very important. ${ }^{5}$

Children represent the future, and ensuring their healthy growth and development ought to be a prime concern of all societies. Children, particularly newborns are vulnerable to malnutrition and infectious diseases, many of which can be effectively prevented or treated. ${ }^{6}$

\section{MATERIALS AND METHODS}

\section{Study Setting}

A prospective observational study conducted in the outpatient department and in-patient wards of Department of Paediatrics, Dr. B.R. Ambedkar Medical College and Hospital, Bangalore.

\section{Study Materials}

\section{Patient Data Collection Form}

Data was collected by using a self-designed data collection form, which consists of details like patient demographics, laboratory data, literacy of the patient's family, drug therapy and other relevant information.

\section{Patient Assent form}

Data was collected by using a self-designed patient consent form.

\section{Patient Medical Record}

Data was collected from Patient Medical Record which comprised of patient demographics, history of patient, general physical examination, laboratory data, and drug therapy.

\section{Study Procedure}

All patients admitted to the wards, PICU, NICU as well as out-patients were reviewed every day. The by-stander of the patients were informed the details of the study and consent was obtained. Patients who met the study criteria were included in the study and data were collected, including age, gender, supporting symptoms, past history, vitals, and therapeutic data including dose, frequency, route of administration, time of administration. Number of antibiotics used and their dosage form were also focused on. The relevant data were collected and documented in the patient data collection form. The work was documented in MS Excel sheet.

\section{RESULT AND DISCUSSION}

A total of 80 patients were reviewed who had Lower Respiratory Tract Infections. 


\section{Age Distribution of Patients}

Out of the 80 patients (see Table 1), 74 (92.5\%) were found be in the age range of 0 to 5 years while $6(7.5 \%)$ patients fell under 5-10 years. No LRTI cases were reported from 11-17 years throughout the study period. A study by Francisco $A D$ et al, ${ }^{7}$ evaluated the risk factors for death due to acute lower respiratory tract infections (ALRTI) in young children. The findings are similar to his study and revealed that children aged $<2$ years were thought to have died from ALRTI. Comparison of cases and live controls suggested that exposure to smoke during cooking, parental smoking and exclusive, prolonged breastfeeding were associated with an increased risk of death from ALRTI. Hence, the lifestyle of a child itself is a risk factor for acquiring a respiratory tract infection.

\section{Gender Distribution}

As in (Table 1), Age ranging from $0-5$ years had 42 $(52.5 \%)$ male and $32(40 \%)$ female, whereas in 6-11 years there were $4(5 \%)$ male and $2(2.5 \%)$ female.There is a significant gender difference in the development, course and outcome of RTIs. Compared to female, male children seem to suffer more commonly from most types of RTIs. In addition, such infections take a more severe course in males, leading to higher mortality. Hence, clinicians should be aware of these differences and take them under consideration when managing patients with RTIs. ${ }^{8}$

\section{Distribution of Patients in IP/OP}

The number of patients in IP was $45(56.25 \%)$ and the patients who attended OP was 35 (43.75\%) (Figure 1).

\section{Monthly Incidence of Infections in IP \& OP Cases}

As evident from (Table 2), throughout the data collection period, it was observed that, majority of the LRTI cases were seen in the month of January, considering both IP and OP. It was 27 and 15 respectively. In February, the number of cases obtained were less compared to December and January.

\section{Visit Types}

In (Table 2), it was found that LRTI cases were admitted to the hospital depending upon the severity of the infection and considering the monitoring parameters as well. In the month of February, $86 \%$ of the patients were seen visiting OP whereas in December 67\% were admitted to the Hospital. Very few patients were admitted in February, which was $14 \%$. Acute respiratory infections are very common and account for a large number of population. According to Floyd MD et al, ${ }^{9}$ many reports based on studies in hospitalized, severely ill chil- dren have added immensely to our knowledge, but most children with LRTI are not ill enough to require hospitalization. The findings supports his study. He added that hospitalization should be based on assessment of cause, incidence, associated agents and seasonal occurrence.

\section{Presence of Characteristic Symptoms}

In the study period it was found that 31 (38.75) patients did not have any characteristic symptom. Maximum patients were found to be presenting with wheezing which was $21(26.25 \%)$. Greenish sputum was seen to be the symptom in only $1(1.25 \%)$ patient. The list of supporting characteristic symptoms are given in Table 3.

\section{Infectious Agents}

The study depicted that most of the cases were caused by bacterial, $78(98 \%)$ whereas only $2(2 \%)$ patients were seen to have viral LRTI. (Figure 2).

\section{TREATMENT MODALITIES}

Antibacterial was used for the treatment of Lower Respiratory Tract Infections. These were either given alone or in combination. Supportive treatment was also given.

1. Patients receiving antibacterial exclusively: It was found that only $1(1.25 \%)$ patient was exclusively on an antibacterial.

2. Patients receiving both antibacterial therapy as well as supportive therapy: Most of the patients received a supportive treatment along with antibacterial therapy and the number of patients receiving both at a time were $63(78.75 \%)$.

3. Patients receiving only supportive treatment: Only $16(20 \%)$ were receiving only a supportive treatment in the study period. They were on symptomatic treatment.

\section{ANTIBACTERIAL THERAPY}

Most of the patients $64(80 \%)$ received antibacterial agents and $16(20 \%)$ patients did not receive it at all. Katherine $\mathrm{L}$ et $a l,{ }^{10}$ stated that millions of courses of antibiotic are prescribed for children with acute cough illness each year, despite evidence from randomized, placebo-controlled trials that such treatments are not effective. Evidence that children with cough $\leq 10$ days should not be treated with antimicrobial agents. Older children with prolonged cough or those with underlying lung diseases may benefit from such therapy. In the study, most of the patients were receiving antibacterials. 


\begin{tabular}{|c|c|c|}
\hline \multicolumn{3}{|c|}{ Demographics of patients } \\
\hline \multicolumn{3}{|c|}{ Age distribution } \\
\hline Age in years & & Number of cases \\
\hline $0-5$ & & 74 \\
\hline $6-10$ & & 6 \\
\hline \multicolumn{3}{|c|}{ Gender distribution } \\
\hline Age range & Males & Females \\
\hline $0-5$ years & 42 & 32 \\
\hline $6-10$ years & 4 & 2 \\
\hline
\end{tabular}

\begin{tabular}{lcccc}
$\begin{array}{l}\text { Table 2: Number of cases collected in IP/OP and the } \\
\text { visit types }\end{array}$ & $\begin{array}{l}\text { List of infections with } \\
\text { respect to months in } \\
\text { IP \& OP }\end{array}$ & Visit Types \\
\cline { 2 - 5 } Months & \multicolumn{4}{c}{ LRTI } \\
\cline { 2 - 5 } & IP & OP & IP\% & OP\% \\
\hline December & 16 & 8 & 67 & 33 \\
January & 27 & 15 & 64 & 36 \\
February & 2 & 12 & 14 & 86 \\
Overall & 45 & 35 & 56.2 & 43.8 \\
\hline
\end{tabular}

Table 3: List of the Presence of Characteristic Symptoms

\begin{tabular}{ccc} 
& Number of cases & Percentage \\
\hline Without any symptoms & 31 & $38.75 \%$ \\
Wheeze & 21 & $26.25 \%$ \\
Rhonchi & 13 & $16.25 \%$ \\
Creptations & 8 & $10 \%$ \\
Conducted sounds & 6 & $7.5 \%$ \\
Greenish sputum & 1 & $1.2 \%$ \\
Total & 80 & $100 \%$ \\
\hline
\end{tabular}

\begin{tabular}{|ccc|}
\hline Table 4: List of Antibacterials Used & \\
\hline Antibacterial used & $\begin{array}{c}\text { Number of } \\
\text { patients }\end{array}$ & Percentage \\
\hline Amoxicillin-clavulenic acid & 30 & $37.5 \%$ \\
Ceftriaxone & 13 & $16.25 \%$ \\
Cefotaxime & 13 & $16.25 \%$ \\
Amikacin & 10 & $12.5 \%$ \\
Azithromycin & 2 & $2.5 \%$ \\
Cefuroxime & 2 & $2.5 \%$ \\
Cephalexin & 2 & $2.5 \%$ \\
Cefixime & 1 & $1.25 \%$ \\
\hline
\end{tabular}

\section{Table 5: Dosage Form of Antibacterial}

\begin{tabular}{ccc}
\hline Dosage form & No. of patients & Percentage \\
\hline Injectable & 55 & 68.75 \\
Syrup & 18 & $22.5 \%$ \\
Tablet & 6 & $7.5 \%$ \\
Drops & 1 & $1.25 \%$ \\
\hline
\end{tabular}

\begin{tabular}{ccc}
\hline $\begin{array}{c}\text { Table 6: List of Supportive Agents Used } \\
\text { Supportive agents }\end{array}$ & No. of patients & Percentage \\
\hline Nebulization & 41 & $27.70 \%$ \\
Cough \& Cold preparation & 40 & $27.02 \%$ \\
Analgesic & 38 & $25.67 \%$ \\
Decongestant & 12 & $8.10 \%$ \\
Antihistamine & 6 & $4.05 \%$ \\
Mucolytic & 3 & $2.02 \%$ \\
Bronchodilator & 1 & $0.67 \%$ \\
Leukotriene antagonist & 1 & $0.67 \%$ \\
\hline
\end{tabular}

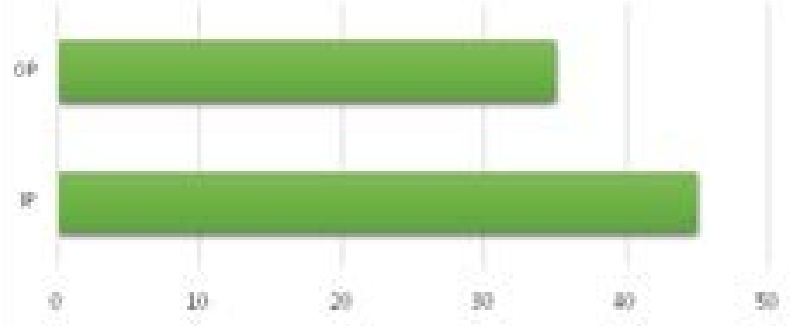

Figure 1: Distribution of Patients in IP/OP

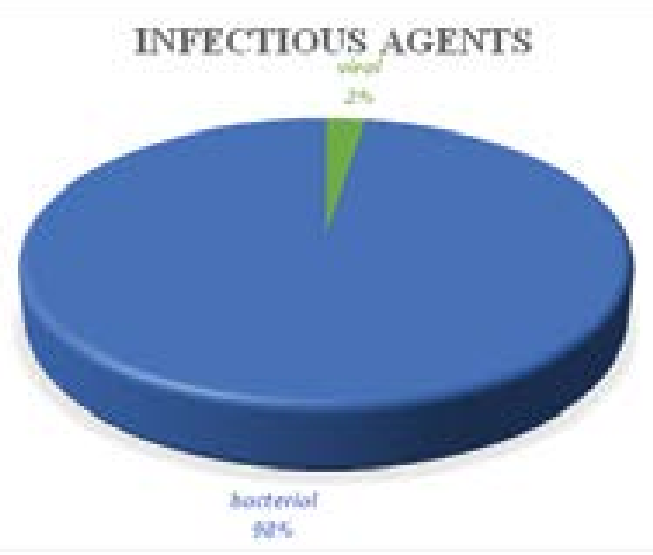

Figure 2: Infectious Agents

\section{Antibacterials Used}

In most of the patients, 30 (37.5\%) Amoxicillinclavulanic acid combination was used followed by cefotaxime and ceftriaxone being used in 13 (16.25\%) patients. The least used was cefixime in 1 (1.25\%) patient. The list of various antibacterials used for the treatment of LRTI cases are given below, in (Table 4).

\section{DOSAGE FORMS USED}

In (Table 5), as most of the patients were admitted to the hospital, the most commonly used dosage form was injectable and was used in 55 patients. Syrup was Indian Journal of Pharmacy Practice, Vol 9, Issue 1, Jan-Mar, 2016 
the second common form used in 18 patients. Only 1 patient received drops.

\section{SUPPORTIVE THERAPY}

In 80 patients, $79(98.75 \%)$ received supportive treatment whereas only $1(1.25 \%)$ patient was exclusively under antibacterial.

\section{SUPPORTIVE AGENTS USED}

Various supportive agents were used along with the antibacterial therapy. In these nebulization agents were used the most, in $41(27.70 \%)$ patients followed by cough and cold preparations in $40(27.02 \%)$ patients. The least used agents were bronchodilator and leukotriene antagonist. Both were given to only $1(0.67 \%)$ patient (Table 6).

This was a seasonal study in which the patients presenting with LRTI were assessed for the symptomatic character, use of antibacterials, its dosage form, visit types, incidence of infection and the supportive treatment given. A total of 80 patients met with the study criteria. Among them $92.5 \%$ were found be in the age range of 0 to 5 years while $7.5 \%$ patients were under $6-10$ years. More number of cases were obtained in the former age group than the latter. Males were affected more than females. There were 42 males and 32 females in the former age group whereas only 4 males and 2 females were affected in the latter. Hence it is concluded that the children at their tender age are prone to respiratory tract infections which could be due to the climatic changes or the poor lifestyle they are born into.

As the LRTI patients had to be treated based on the severity of the symptoms and monitoring of their respiratory health was desired, most of the patients were encouraged for hospital stay in view of patient safety. This is evident from the data that $56.25 \%$ of the total were admitted to the In-Patient ward and the patients who visited OP was $43.75 \%$.

Majority of the LRTI cases were seen in the month of January, considering both IP and OP. In IP it was $33.75 \%$ and only $18.75 \%$ were obtained in OP. The least number of cases were obtained in February. In the month of February, $86 \%$ of the patients were seen visiting OP whereas in December 67\% were admitted to the Hospital. Very few patients were admitted in February, which was $14 \%$.

Wheezing was the most commonly seen characteristic symptom supporting the infection and was seen in $26.25 \%$ of patients. Greenish sputum was seen to be the symptom in only $1(1.25 \%)$ patient.

Bacteria was the most common causative organism in LRTI. It was found that the maximum number, $97.5 \%$ were affected by bacterial infection. Only $2.5 \%$ of patients were seen to have viral LRTI.

The use of an antibacterial was a very necessary step in the management of the infection. However, the treatment modality varied in different patients. Considering the same fact, the entire data can be categorized into three;

- Patients exclusively under antibacterial therapy.

- Patients receiving supportive therapy along with antibacterial treatment.

- Patients receiving only supportive therapy.

Most of the patients, $80 \%$ received an antibacterial whereas only $16 \%$ did not receive any. Amoxicillinclavulenic acid combination was used in maximum number, $37.5 \%$ of patients followed by cefotaxime and ceftriaxone, which was $16.25 \%$. cefixime was used only in $1.25 \%$ of patient.

Supportive therapy was received by $98.75 \%$ of patients while $1.25 \%$ did not receive. Various agents were used along with the antibacterial for the maintaining the proper respiratory health of the patients. Among them, nebulization agents were used the most, in $27.70 \%$ patients followed by cough and cold preparations which was $27.02 \%$. The least used agents were bronchodilator and leukotriene antagonist. Both were given to only $0.67 \%$ of Patient.

As most of the patients were admitted to the hospital, the most commonly used dosage form was injectable and was used in 55 patients. Syrup was the second common form used in 18 patients. Only 1 patient received drops.

\section{CONCLUSION}

Antibacterial combination is more preferred in the present scenario. This shows the development of resistance due to the reckless use of the antibacterials without even considering the culture sensitivity tests. Viral infection is self-limiting and does not require to be treated with an antibacterial. So, it is evident that judicious use of such therapeutic agents are necessary. Otherwise, the children developing resistance would be difficult to be treated in later life as the normal doses will be ineffective in them resulting in poor prognosis. This would not only affect their quality of life but also the economic values. There are various other dreadful infectious diseases which would affect them later. Injudicious use of antibacterials at early stages can lead to serious consequences where mitigation of the disease would turn questionable. The more they are affected by the infections, the more they are subjected to adopt such inutile treatment options. Children are the powerful youth of tomorrow, a healthy generation gives a wealthy nation. 


\section{ACKNOWLEDGEMENTS}

The authors would like to thank Mr. Premnath Reddy, Chairman and Dr. Divakar Goli, Principal of Acharya and B. M. Reddy College of Pharmacy for their unending support and for providing excellent platform for conducting this study.

Also we would like to thank Dr. Vishnupriya, Department of Pediatrics, B. R. Ambedkar Medical College and Hospital for her contribution to the manuscript.

\section{CONFLICT OF INTEREST}

There is no conflict of interest by author on the manuscript.

\section{ABBREVIATION USED}

IP: In-patient

OP: $\quad$ Out-patient

RTIs: Respiratory Tract infections.

LRTIs: $\quad$ Lower Respiratory Tract Infections

\section{REFERENCES}

1. Respiratory Tract Infections [Internet]. 2012 Apr 20 [cited 2015 Aug 17]. Available from: http://www.nps.org.au/conditions/respiratory-problems/respiratorytract-infections.

2. Mike. S. The use of antibacterials in children: a report of the Specialist Advisory Committee on Antimicrobial Resistance (SACAR) Paediatric Subgroup. London. 2015;60(1):i15-i26.

3. Franz. A. Correlation of viral load of respiratory pathogens and co-infections with disease severity in children hospitalized for lower respiratory tract infection. Journal of Clinical Virology. 2010;48(4):239-45.

4. Marlow D, Redding B. Textbook of Pediatric Nursing. $6^{\text {th }}$ ed. Philadelphia: Elsevier; 1988.

5. Lima. R. Do risk factors for childhood infections and malnutrition protect against asthma? A study of Brazilian Male Adolescents. American Journal of Public Health. 2003;93(11):1858-64.

6. World Health Organisation- Child Health. [Internet]. [cited 2015 Aug 21]. Available from: http://www.who.int/topics/child_health/en/

7. Francisco AD. Risk Factors for Mortality from Acute Lower Respiratory Tract Infections in Young Gambian Children. Bangladesh.1993;22(6):1174-82.

8. Mathew. E. sex differences in the incidence and severity of respiratory tract infections. Respiratory Medicine. 2007;101(9):1845-63.

9. Floyd. MD. Acute Lower Respiratory Tract Infections in non-hospitalized children. Journal of Pediatrics.1986;108(5):635-46.

10. Katherine. L. Cough illness/Bronchitis-Principles of Judicious Use of Antimicrobial Agents. Pediatrics.1998;101(sup 1):178-81. 